\title{
The current status of quantitative SPECT/CT in the assessment of transthyretin cardiac amyloidosis
}

\author{
Stuart C. Ramsay, MBBS, MD, FRACP, FAANMS, DDU, ${ }^{a, b}$ and Claire Cuscaden, \\ MBBS, FRANZCR ${ }^{\mathrm{c}, \mathrm{d}}$ \\ a Nuclear Medicine and PET, Royal Brisbane and Women's Hospital, Herston, QLD, Australia \\ b School of Medicine, James Cook University, Townsville, Australia \\ c Department of Medical Imaging, Princess Alexandra Hospital, Wooloongabba, QLD, Australia \\ d The University of Queensland, St Lucia, QLD, Australia
}

Received Oct 7, 2019; accepted Oct 7, 2019

doi:10.1007/s12350-019-01935-1

\begin{abstract}
Nuclear medicine bone scans differentiate ATTR cardiomyopathy (ATTR-CM) from light chain cardiac amyloidosis and other myocardial disorders, helping to make the diagnosis without biopsy. Standard bone scans are not absolutely quantitative, so are assessed by comparing the heart to other tissues. The standard visual scoring system compares heart to bone. This accurately diagnoses ATTR-CM and has been validated in a multicenter study, but has limitations. Semiquantitative techniques including heart/contralateral thorax (H/CL) and heart/whole body ratio (H/WB) improve on visual scoring but still rely on extracardiac sites as comparators. Absolute quantitation of myocardial uptake using quantitative SPECT should help overcome these shortcomings. In ATTR-CM, this technique is practical, accurately makes the diagnosis and provides information that is not identical to visual scores. However, more work needs to be done. The reproducibility in ATTR-CM must be tested. Larger studies need to be undertaken to determine whether quantitative SPECT measurements can assess prognosis, disease progression or treatment response. As ATTR-CM is relatively uncommon multicenter trials will help recruit enough subjects to answer these questions. Accurate measurement techniques are needed in ATTR-CM to enable appropriate use of proven therapy and to conduct trials of new therapeutic agents. Quantitative bone scans offer a promising avenue.
\end{abstract}

Key Words: Transthyretin cardiac amyloidosis • Bone scan • Quantitation

\section{INTRODUCTION}

Transthyretin (ATTR) cardiac amyloidosis is associated with heart failure. ${ }^{1}$ Nuclear medicine bone scans differentiate ATTR cardiac amyloidosis from light chain (AL) cardiac amyloidosis and other myocardial

See related article, pp. 1456-1463

Reprint requests: Stuart C. Ramsay, MBBS, MD, FRACP, FAANMS, DDU, Nuclear Medicine and PET, Royal Brisbane and Women's Hospital, Herston, QLD, Australia; stuart.ramsay1@jcu.edu.au

J Nucl Cardiol 2020;27:1464-8.

$1071-3581 / \$ 34.00$

Copyright (c) 2019 American Society of Nuclear Cardiology. disorders with increased left ventricular wall thickness. ${ }^{2-4}$ In appropriately selected individuals, bone scans can diagnose ATTR cardiomyopathy (ATTR-CM) without endomyocardial biopsy. ${ }^{1,5}$ The technetium $99 \mathrm{~m}(\mathrm{Tc})$ labeled phosphate-based agents hydroxymethylene diphosphonate (HMDP), pyrophosphate (PYP) and 3,3diphosphono-1,2-propanodicarboxylic acid (DPD) have equivalent diagnostic accuracy for detecting ATTR$\mathrm{CM}^{2,6,7}$ though it is worth remembering that the images produced by these different agents are not identical. ${ }^{8}$ Given recent developments in bone scan imaging technology it is timely to consider how best to report cardiac bone scans. 


\section{VISUAL SCORING}

Standard bone scans are not absolutely quantitative, so cardiac images are assessed using relative indices, with heart uptake being compared to other tissues. The most widely used index is a visual comparison of cardiac uptake to bone giving scores $0-3$ with 0 representing no myocardial disease. ${ }^{9}$ This accurately identifies ATTR-CM ${ }^{9,10}$ and has been validated in a multicenter study. ${ }^{2}$ But there are limitations. The bone that acts as the comparator has not been constant across reported studies, producing reporter and referrer confusion. This has been addressed in a recent expert consensus, with a comparison of myocardium to rib being defined as standard. ${ }^{11}$ Although visual scores distinguish 2 groups with clearly different prognoses (those with ATTR-CM and those without), they have not proven useful in risk stratification of individuals with proven ATTR-CM. ${ }^{10,12}$ Given that prognosis in wtATTR, the most common subtype of ATTR, is determined by the stage of cardiac involvement rather than the nature of extracardiac disease ${ }^{13}$ why isn't this reflected in the visual score?

ATTR is a systemic disease and abnormal protein is deposited at sites other than myocardium, so bone scan uptake can be seen at extracardiac sites. In ATTR related to genetic mutations (mATTR), the extracardiac sites are determined by the syndrome that accompanies the specific mutation, with neural involvement being common and preceding cardiac involvement in some subtypes. ${ }^{14}$ In ATTR with normal wild type genotype (wtATTR), neural involvement is not typically part of the clinical syndrome. The increased incidence of carpal tunnel syndrome in wtATTR appears to relate to ATTR deposition in tenosynovial tissues. ${ }^{15}$ Synovial deposition also probably plays a role in large joint arthropathy: individuals with wtATTR have an increased rate of knee and hip replacements. ${ }^{16}$ Tendinopathy with ATTR deposition is associated with an increased risk of spontaneous biceps tendon rupture. ${ }^{17}$ Skeletal muscle uptake can be intense with DPD, ${ }^{18}$ but appears to be less marked with HMDP (personal observation) and is not a feature of PYP scans. ${ }^{19}$ Bone scan uptake in fat is variably seen, partly because amyloid fat deposition varies between subtypes of amyloidosis: amyloid is present in abdominal fat biopsy in $84 \%$ of cardiac AL, $45 \%$ of mATTR-CM but only $15 \%$ of wtATTR-CM. ${ }^{20}$ Deposition of ATTR occurs in the lungs and can cause bone scan uptake. ${ }^{21}$ In wtATTR, unlike in AL, specific renal involvement with amyloid is uncommon. ${ }^{22} \mathrm{Hepa}-$ tic uptake can occur in mATTR ${ }^{23}$ but is not a common feature of bone scans in wtATTR (personal observation).

In practice, the degree of apparent reduction in bone uptake, the main criterion used to distinguish grade 2 from grade $3^{11}$, might relate to having such intense cardiac uptake that activity is directed away from the skeleton, and hence represent severe myocardial disease. However, this is not the only possible explanation. Apparently, reduced skeletal uptake might also be because a high level of uptake at extracardiac disease sites has reduced availability of radiopharmaceutical for uptake in the skeleton. It could even relate to a thresholding issue-when there is intense extracardiac uptake (especially with intense musculotendinous uptake around shoulders and/or hips) the images may be thresholded to this intense uptake making the bones less discernible. This means that a patient may be allocated to grade 3 rather than 2 because of the degree of extracardiac disease rather than just the amount of uptake in the heart.

The mechanism by which bone scan radiopharmaceuticals are taken up and retained by the myocardium remains incompletely understood, ${ }^{23}$ but it remains a reasonable hypothesis that more cardiac amyloid deposition will be associated with more bone scan uptake, that a greater amount of amyloid deposition is associated with a worse prognosis, and that changes in the amyloid burden can be assessed by changes in the amount of radiopharmaceutical uptake. The fact that that there is no difference in prognosis between visual grades 2 and 3 is disappointing, but is likely to be because the differentiation between these scores is determined by the amount of extracardiac ATTR deposition (which does not significantly affect prognosis) as well as by the amount of cardiac ATTR.

\section{SEMIQUANTITATIVE INDICES}

Attempts have been made to improve measurement of bone scan uptake with semiquantitative indices. Heart to contralateral thorax $(\mathrm{H} / \mathrm{CL})$ has been validated for 1 and 3 hour PYP scans in a multicenter study. ${ }^{24}$ This study suggested $\mathrm{H} / \mathrm{CL}$ has prognostic significance, however, there was only a small numerical difference between the cutoff value for diagnosis of ATTR and that related to poorer prognosis. For HMDP scans, Heart/ Whole Body ratio (H/WB) improves on the visual score, and is more reliable than $\mathrm{H} / \mathrm{CL}^{25}$ but its role in prognosis is not yet clear. Thus, semiquantitative techniques can improve on visual scores, but they still rely on extracardiac sites as comparators. This can be a problem when there is abnormal extracardiac uptake, whether this is caused by ATTR or other disease. For example, $\mathrm{H} / \mathrm{CL}$ will be affected by uptake related to ATTR lung involvement, and with H/WB there can be error due to variation in musculoskeletal uptake. For example, wtATTR-CM tends to occur in older males 
who are more likely to have intercurrent osteoblastic bone disease such as prostate cancer metastases, and the associated increased skeletal uptake causes an underestimate of H/WB compared to those with normal skeletons. The relative nature of these techniques also reduces accuracy in monitoring disease progression or treatment response due to variation in uptake in the comparator tissue over time-for example with prostate metastases skeletal uptake will vary with time depending on treatment - changing H/WB even when cardiac uptake is stable.

\section{PET AND SPECT ABSOLUTE QUANTITATION}

Absolute quantitation of myocardial uptake should help overcome these shortcomings. Quantitation can be achieved with PET, but to date the most commonly used PET bone scan agent, NaF, has not proven as useful in the assessment of cardiac ATTR as single-photon radiopharmaceuticals. ${ }^{26}$ PET-based agents that image amyloid proteins can provide images of cardiac amyloid. ${ }^{27}$ However, to date, studies using these agents are preliminary and have not yet provided information in ATTR-CM that has been shown to directly affect patient care, partly perhaps because PET is not as readily available as some other imaging techniques.

Another promising technique is quantitative SPECT which is now commercially available. The clinical adoption of this technique was initially limited by a number of technical challenges ${ }^{28}$. Phantom studies and technical advances have addressed many of these issues $^{29}$, and the technique is now making its way into clinical use. Quantitative SPECT images are reconstructed using commercially available softwareincorporating CT-based attenuation correction, scatter correction and an appropriate iterative reconstruction technique. As with PET, the images represent parametric maps of radiopharmaceutical distribution with units of $\mathrm{kBq} / \mathrm{mL}$ standardized to the time of injection, and can be corrected for injected dose and volume of distribution to give SUVs. Quantitative SPECT images can be displayed in conjunction with the CT component for anatomic localisation. In individuals without cardiac amyloidosis myocardial uptake is less intense than, or indistinguishable from blood pool on bone scan SPECT images. ${ }^{30}$ VOIs can be drawn on the SPECT images, or on the CT images and then projected onto the SPECT images, allowing measurement of SUVs within various structures including myocardium, blood pool and individual bones.

Further assessment of cardiac disease burden can be undertaken by measuring cardiac metabolic activity (CMA) and cardiac metabolic volume (CMV) which can be calculated from quantitative SPECT images using standard algorithms that identify voxels with uptake above certain thresholds within a defined volume of interest using techniques initially developed for total lesion glycolysis (TLG) and metabolic tumor volume (MTV) in oncology (Figure 1). These measurements have proven useful in FDG PET assessment of cardiac sarcoidosis $^{31}$ and would appear to carry promise for measuring cardiac amyloid burden. CMA and MTV are most reliably calculated when cardiac uptake is high, and so hold promise in assessing patients with known ATTR based solely on their cardiac uptake, offering a technical solution to the previously discussed grade 2 versus 3 issue. When there is no (or very low grade) myocardial uptake these algorithms are less accurate in defining the myocardium, hence are likely to be less useful in making the diagnosis of ATTR-CM.

To date, few published studies have used quantitative SPECT in ATTR-CM. In a small single-center study using HMDP, quantitative SPECT derived myocardial SUVmax accurately differentiated ATTR-CM from other cardiac diseases and this measurement was found to be sufficiently robust that a reference range for individuals without cardiac involvement could be developed, against which range a single potentially affected

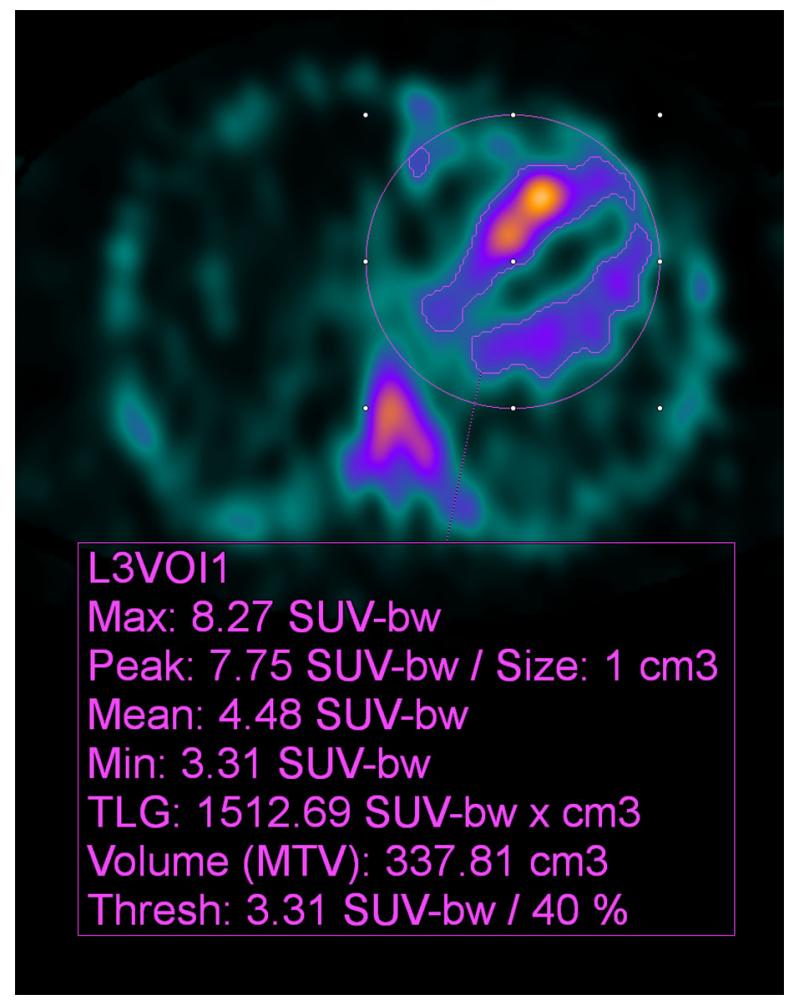

Figure 1. Transaxial quantitative SPECT image of the thorax showing sample measurements of myocardial uptake of HMDP ( $T L G$ total lesion glycoloysis = cardiac metabolic activity, MTV metabolic tumor volume = cardiac metabolic volume). 
individual could be compared ${ }^{30}$. Caobelli et al. have now expanded on this preliminary work by showing that quantitative cardiac SPECT using DPD is practical, can make the diagnosis of ATTR-CM, and provides information that is not identical to visual score in grade 2 and 3 patients. $^{32}$

\section{THE FUTURE OF QUANTITATIVE BONE SCANS}

More work needs to be done in this field. In quantitative SPECT, a regular QC process incorporating a NIST traceable calibration source can ensure measurement stability over time, and is a good first step in ensuring that different sites make equivalent measurements. It has also been proposed that quantitative SPECT should be subject to the same NEMA NU 2 QC process as PET. ${ }^{33}$ If quantitative SPECT is to be used for progress assessment the reproducibility of measurements in individuals with ATTR-CM must be assessed. Larger clinical trials need to be undertaken to determine whether quantitative SPECT measurements can provide prognostic information in ATTR or play a role in assessing disease progression and treatment response. However, ATTR-CM is a relatively uncommon disease, so it is unlikely that a single site will rapidly obtain data from enough patients to conduct conclusive studies. Multicenter cooperative efforts are likely to be the best way to find answers to important clinical questions in ATTR-CM, including the role of quantitative SPECT.

Tafamidis has been proven to be efficacious in ATTR-CM ${ }^{34}$ and treatments designed to reduce amyloid burden are in development. A recent Australian study looking at incidental cardiac uptake on bone scans performed for non-cardiac purposes suggested a prevalence of ATTR-CM of $4.2 \%$ in men aged 85 and over ${ }^{35}$ confirming that ATTR-CM, and wtATTR-CM in particular, are not as uncommon as was previously believed, and are likely to be seen more frequently as the population ages. To meet the needs of these patients we need to develop readily accessible techniques that accurately identify these individuals in the community, and can assess their progress, to enable appropriate use of proven therapy and to assist in conducting dedicated trials of new pharmacological agents. Quantitative bone scans offer a promising avenue.

\section{Disclosure}

Dr. Ramsay acts as an unpaid advisor to Pfizer on cardiac imaging. He has no other potential conflicts of interest. Dr. Cuscaden has no conflicts of interest to declare.

\section{References}

1. Maurer MS, Elliott P, Comenzo R, Semigran M, Rapezzi C. Addressing common questions encountered in the diagnosis and management of cardiac amyloidosis. Circulation 2017;135(14):1357-77.

2. Gillmore JD, Maurer MS, Falk RH, Merlini G, Damy T, Dispenzieri A, et al. Nonbiopsy diagnosis of cardiac transthyretin amyloidosis. Circulation 2016;133(24):2404-12.

3. Cappelli F, Gallini C, Di Mario C, Egidio Natalino Costanzo, Luca Vaggelli, Francesca Tutino, et al. Accuracy of 99mTc-Hydroxymethylene diphosphonate scintigraphy for diagnosis of transthyretin cardiac amyloidosis. J Nucl Cardiol 2017. https://doi. org/10.1007/s12350-017-0922-Z

4. Galat A, Rosso J, Guellich A, Van Der Gucht A, Rappeneau S, Bodez D, et al. Usefulness of (99m)Tc-HMDP scintigraphy for the etiologic diagnosis and prognosis of cardiac amyloidosis. Amyloid 2015;22(4):210-20.

5. Habib G, Bucciarelli-Ducci C, Caforio ALP, Cardim N, Charron $\mathrm{P}$, Cosyns B, et al. Multimodality imaging in restrictive cardiomyopathies: An EACVI expert consensus document In collaboration with the "Working Group on myocardial and pericardial diseases" of the European Society of Cardiology Endorsed by The Indian Academy of Echocardiography. Eur Heart J Cardiovasc Imaging 2017;18(10):1090-121.

6. Andrikopoulou E, Bhambhvani P. Nuclear imaging of cardiac amyloidosis. J Nucl Cardiol 2017.

7. Treglia G, Glaudemans A, Bertagna F, Hazenberg BPC, Erba PA, Giubbini R, et al. Diagnostic accuracy of bone scintigraphy in the assessment of cardiac transthyretin-related amyloidosis: A bivariate meta-analysis. Eur $\mathbf{J}$ Nucl Med Mol Imaging 2018;45(11):1945-55.

8. Rapezzi C, Gagliardi C, Milandri A. Analogies and disparities among scintigraphic bone tracers in the diagnosis of cardiac and non-cardiac ATTR amyloidosis. J Nucl Cardiol 2018.

9. Perugini E, Guidalotti PL, Salvi F, Cooke RM, Pettinato C, Riva $\mathrm{L}$, et al. Noninvasive etiologic diagnosis of cardiac amyloidosis using 99mTc-3,3-diphosphono-1,2-propanodicarboxylic acid scintigraphy. J Am Coll Cardiol 2005;46(6):1076-84.

10. Hutt DF, Fontana M, Burniston M, Quigley AM, Petrie A, Ross JC, et al. Prognostic utility of the Perugini grading of $99 \mathrm{mTc}-\mathrm{DPD}$ scintigraphy in transthyretin (ATTR) amyloidosis and its relationship with skeletal muscle and soft tissue amyloid. Eur Heart J Cardiovasc Imaging 2017;18(12):1344-50.

11. Dorbala S, Ando Y, Bokhari S, Dispenzieri A, Falk RH, Ferrari VA, et al. ASNC/AHA/ASE/EANM/HFSA/ISA/SCMR/SNMMI expert consensus recommendations for multimodality imaging in cardiac amyloidosis: Part 1 of 2-evidence base and standardized methods of imaging. J Nucl Cardiol 2019.

12. Vranian MN, Sperry BW, Hanna M, Hachamovitch R, Ikram A, Brunken RC, et al. Technetium pyrophosphate uptake in transthyretin cardiac amyloidosis: Associations with echocardiographic disease severity and outcomes. J Nucl Cardiol 2018;25(4):124756.

13. Pinney JH, Whelan CJ, Petrie A, Dungu J, Banypersad SM, Sattianayagam $\mathrm{P}$, et al. Senile systemic amyloidosis: Clinical features at presentation and outcome. J Am Heart Assoc 2013;2(2):e000098.

14. Kapoor M, Rossor AM, Laura M, Reilly MM. Clinical presentation, diagnosis and treatment of TTR amyloidosis. J Neuromuscul Dis 2019;6(2):189-99. 
15. Sekijima $Y$, Uchiyama $S$, Tojo K, Sano K, Shimizu Y, Imaeda T, et al. High prevalence of wild-type transthyretin deposition in patients with idiopathic carpal tunnel syndrome: A common cause of carpal tunnel syndrome in the elderly. Hum Pathol 2011;42(11):1785-91.

16. Rubin J, Alvarez J, Teruya S, Castano A, Lehman RA, Weidenbaum M, et al. Hip and knee arthroplasty are common among patients with transthyretin cardiac amyloidosis, occurring years before cardiac amyloid diagnosis: Can we identify affected patients earlier? Amyloid 2017;24(4):226-30.

17. Geller HI, Singh A, Alexander KM, Mirto TM, Falk RH. Association between ruptured distal biceps tendon and wild-type transthyretin cardiac amyloidosis. JAMA 2017;318(10):962-3.

18. Ross JC, Hutt DF, Burniston M, Page J, Steeden JA, Gillmore JD, et al. Quantitation of $(99 \mathrm{~m}) \mathrm{Tc}-\mathrm{DPD}$ uptake in patients with transthyretin-related cardiac amyloidosis. Amyloid 2018;25(3):203-10.

19. Sperry BW, Gonzalez MH, Brunken R, Cerqueira MD, Hanna M, Jaber WA. Non-cardiac uptake of technetium-99m pyrophosphate in transthyretin cardiac amyloidosis. J Nucl Cardiol 2018.

20. Quarta CC, Gonzalez-Lopez E, Gilbertson JA, Botcher N, Rowczenio D, Petrie A, et al. Diagnostic sensitivity of abdominal fat aspiration in cardiac amyloidosis. Eur Heart J 2017;38(24):1905-8.

21. Cappelli F, Gallini C, Costanzo EN, Tutino F, Ciaccio A, Vaggelli $\mathrm{L}$, et al. Lung uptake during $99 \mathrm{mTc}$-hydroxymethylene diphosphonate scintigraphy in patient with TTR cardiac amyloidosis: An underestimated phenomenon. Int J Cardiol 2018;254:346-50.

22. Dember LM. Amyloidosis-associated kidney disease. J Am Soc Nephrol 2006;17(12):3458-71.

23. Singh V, Falk R, Di Carli MF, Kijewski M, Rapezzi C, Dorbala S. State-of-the-art radionuclide imaging in cardiac transthyretin amyloidosis. J Nucl Cardiol 2019;26(1):158-73.

24. Castano A, Haq M, Narotsky DL, Goldsmith J, Weinberg RL, Morgenstern R, et al. Multicenter study of planar Technetium 99m pyrophosphate cardiac imaging: Predicting survival for patients with ATTR cardiac amyloidosis. JAMA Cardiol 2016;1(8):880-9.

25. Gallini C, Tutino F, Martone R, Ciaccio A, Costanzo EN, Taborchi $\mathrm{G}$, et al. Semi-quantitative indices of cardiac uptake in patients with suspected cardiac amyloidosis undergoing 99mTc-HMDP scintigraphy. J Nucl Cardiol 2019.
26. Martineau P, Finnerty V, Giraldeau G, Authier S, Harel F, Pelletier-Galarneau M. Examining the sensitivity of $18 \mathrm{~F}-\mathrm{NaF}$ PET for the imaging of cardiac amyloidosis. J Nucl Cardiol 2019.

27. Kircher M, Ihne S, Brumberg J, Morbach C, Knop S, Kortum KM, et al. Detection of cardiac amyloidosis with (18)F-Florbetaben$\mathrm{PET} / \mathrm{CT}$ in comparison to echocardiography, cardiac MRI and DPD-scintigraphy. Eur J Nucl Med Mol Imaging 2019;46(7):1407-16.

28. Bailey DL, Willowson KP. Quantitative SPECT/CT: SPECT joins PET as a quantitative imaging modality. Eur J Nucl Med Mol Imaging 2014;41(Suppl 1):S17-25.

29. Armstrong IS, Hoffmann SA. Activity concentration measurements using a conjugate gradient (Siemens xSPECT) reconstruction algorithm in SPECT/CT. Nucl Med Commun 2016;37(11):1212-7.

30. Ramsay SC, Lindsay K, Fong W, Patford S, Younger J, Atherton J. Tc-HDP quantitative SPECT/CT in transthyretin cardiac amyloid and the development of a reference interval for myocardial uptake in the non-affected population. Eur $\mathrm{J}$ Hybrid Imaging 2018;2(1): 17

31. Ahmadian A, Pawar S, Govender P, Berman J, Ruberg FL, Miller EJ. The response of FDG uptake to immunosuppressive treatment on FDG PET/CT imaging for cardiac sarcoidosis. J Nucl Cardiol 2017;24(2):413-24.

32. Caobelli F, Braun M, Haaf P, Wild D, Zellweger MJ. Quantitative $(99 \mathrm{~m}) \mathrm{Tc}-\mathrm{DPD}$ SPECT/CT in patients with suspected ATTR cardiac amyloidosis: Feasibility and correlation with visual scores. J Nucl Cardiol 2019.

33. Ryu H, Meikle SR, Willowson KP, Eslick EM, Bailey DL. Performance evaluation of quantitative SPECT/CT using NEMA NU 2 PET methodology. Phys Med Biol 2019;64(14):145017.

34. Maurer MS, Schwartz JH, Gundapaneni B, Elliott PM, Merlini G, Waddington-Cruz $\mathrm{M}$, et al. Tafamidis treatment for patients with transthyretin amyloid cardiomyopathy. $\mathrm{N}$ Engl J Med 2018;379(11):1007-16.

35. Cuscaden C, Smith J, Goodwin B, Ramsay S. Prevalence of transthyretin (ATTR) cardiac amyloidosis in the Australian population estimated using 99mTc-hydroxymethylene diphosphonate planar bone scans. Int Med J 2019;49(S2):6.

Publisher's Note Springer Nature remains neutral with regard to jurisdictional claims in published maps and institutional affiliations. 